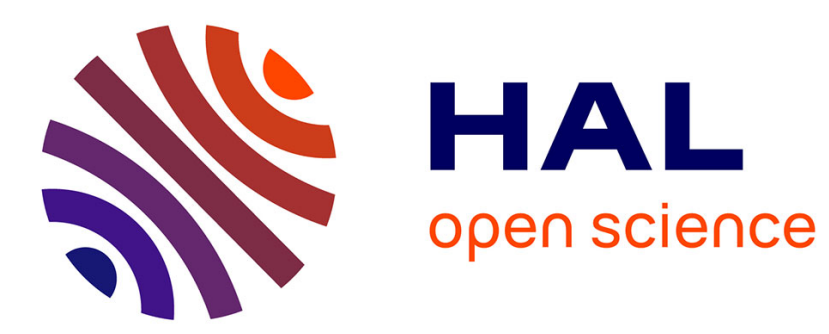

\title{
Influence of the description of the scattering matrix on permittivity reconstruction with a quantitative imaging procedure: polarization effects
}

Christelle Eyraud, Hassan Saleh, Jean-Michel Geffrin

\section{- To cite this version:}

Christelle Eyraud, Hassan Saleh, Jean-Michel Geffrin. Influence of the description of the scattering matrix on permittivity reconstruction with a quantitative imaging procedure: polarization effects. Journal of the Optical Society of America. A, Optics and image science, 2019, 36 (2), pp.234-244. 10.1364/josaa.36.000234 . hal-01986569

\section{HAL Id: hal-01986569 \\ https://hal.science/hal-01986569}

Submitted on 11 Feb 2020

HAL is a multi-disciplinary open access archive for the deposit and dissemination of scientific research documents, whether they are published or not. The documents may come from teaching and research institutions in France or abroad, or from public or private research centers.
L'archive ouverte pluridisciplinaire $\mathbf{H A L}$, est destinée au dépôt et à la diffusion de documents scientifiques de niveau recherche, publiés ou non, émanant des établissements d'enseignement et de recherche français ou étrangers, des laboratoires publics ou privés. 


\title{
Influence of the description of the scattering matrix on the permittivity reconstruction with a quantitative imaging procedure: polarization effects
}

\author{
C. Eyraud ${ }^{1, *}$, H. Saleh ${ }^{1,2}$, J.-M. Geffrin ${ }^{1}$ \\ ${ }^{1}$ Aix Marseille Univ, CNRS, Centrale Marseille, \\ Institut Fresnel, F-13013 Marseille, France \\ 2 Centre Commun de Ressources en Microondes CCRM, \\ 5 rue Enrico Fermi, Marseille, France \\ * Corresponding author: christelle.eyraud@fresnel.f
}

\begin{abstract}
This paper focuses on the role of polarization - and more specifically, the effect of its selection - in 3D quantitative imaging obtained from scattered field measurements. Although polarization is now commonly used in linear imaging procedures (when unknowns are linked by a linear relationship to the measured signal), the influence of polarization choice is generally ignored in non-linear imaging problems. In this paper, we propose a formulation to obtain the 3D permittivity map, by a non-linear imaging procedure, from the scattering matrix. This allows, from the same data set, to select the desired polarization case as input data for the imaging algorithm. We present a study of the influence of the input data polarization choice on the reconstructed permittivity map. This work shows that a suitable basis choice for the description of the scattering matrix and an appropriate selection of the element of this scattering matrix can greatly improve imaging results.
\end{abstract}

\section{INTRODUCTION}

Electromagnetic wave probing is a powerful tool to reach the physical features of a scene in a non destructive way and has a wide area of potential applications in several domains. Indeed, after interaction with the scene, the radiated field contains the characteristics of the targets (position, shape, electromagnetic properties). Considering real measurements, the field can not obviously be measured on an entire surface enclosing the targets, only some directions of the transmitter and of the receiver are possible and the data are thus spatially truncated in such problems. It is crucial to exploit any opportunity to have information on the scene, and an appropriate selection of polarization is one of them.

The polarimetric information of the electromagnetic wave is of great interest for imaging purposes and has been used for several years in radar [1] for radar remote sensing applications [2], [3], and in light scattering [4], [5] such as, for example, for optical biomedical applications [6]. In these last cases, the recorded signal often allows a direct access to an image of the scene. To extract as much information as possible, the recorded signal can be acquired with different polarizations for both the transmitter and the receiver. Indeed, the polarimetric contrast is an interesting way to detect targets [7], [8]. Different methods have thus been developed to optimize the polarimetric contrast between two regions of the observed scene or between the target and the embedding medium [9]. When the recorded signal does not directly provide an image of the scene and when an imaging procedure is required, polarization must be integrated into the imaging process. In the case where unknowns are linearly related, under certain assumptions, to the measured signal, polarization has been used for several years to improve images. This is the case, for example, with synthetic aperture radar (SAR) [10], [11] scattering tomography [12] or Optical Coherence Tomography [13]. These procedures provide "qualitative" maps (except in cases where the targets are weak scatterers), allowing the targets to be detected and their positions and shapes to be obtained.
When one want, in addition, a permittivity map of the imaged area, "quantitative" imaging procedures must be used. In such procedures, a significant difference from the processes described above is that the unknowns (3D permittivity map) are related to the measured data by a non-linear relationship. In these procedures, the influence of polarization is very rarely taken into account. When different polarizations are taken into account, they are generally considered to contain the same amount of information and the processing of each polarization state is performed in the same way [14], [15], [16], [17]. In this contribution, we are interested in quantitative imaging and we propose a formulation to perform this quantitative imaging from the scattering matrix (which can be written in different basis) in order to take polarization into account easily. For 3D objects that have a high degree of freedom, it is all the more necessary to make the most of all the available information and polarization is important information. We propose here a study of the influence of the polarization selection on the reconstructed permittivity map. To focus only on the influence of polarization, no a priori information (neither on the object sought nor on the noise disturbing the measurements) is incorporated into the inversion procedure and no regulation term is added to the cost function. Images are obtained from both calculations and measurements. Linear and circular polarizations are studied. For the different cases, we are interested in the consequence of choosing a given scattering element of the scattering matrix over another and in the choice of the basis for writing the scattering matrix. We show that appropriate choices can significantly improve the reconstructed map.

Part II is devoted to the scattering matrix construction in the various basis and to the presentation of the different polarization cases. The imaging algorithm is explained in part III. The details of the experimental setup and the measurement of the fields are explained in part IV. The imaging results are presented in part $\mathrm{V}$ and a discussion on these results follows in part VI. Some concluding remarks are finally given in part VII. 


\section{SCATTERING MATRIX}

In far field, for a non-absorbing medium, the complex amplitude scattering matrix $\left[\mathbf{S}_{\mathcal{B}}\right]$ describes the relationship between the incident field vector $\left[\mathbf{E}^{\mathbf{i}}{ }_{\mathcal{B}}\right]$ and the scattered field vector $\left[\mathbf{E}_{\mathcal{B}}^{\mathbf{s}}\right][4]$.

$$
\left[\mathbf{E}^{\mathbf{s}}{ }_{\mathcal{B}}\right]=\left[\mathbf{S}_{\mathcal{B}}\right]\left[\mathbf{E}^{\mathbf{i}}{ }_{\mathcal{B}}\right]
$$

This complex amplitude scattering matrix depends on the polarization type (linear, circular, elliptical) and on the choice of the basis $\mathcal{B}$ for this polarization description. Since far-field conditions are assumed, the field vector has no radial component and is then totally determined by its components along the two basis vectors. In a multistatic configuration, this scattering matrix $\left[\mathbf{S}_{\mathcal{B}}\right]$ is defined as follows for each direction of the incident wave vector $\mathbf{k}^{\mathbf{i}}$ and each direction of the scattered wave vector $\mathbf{k}^{\mathbf{s}}$ :

$$
\left(\begin{array}{c}
E_{p}^{s} \\
E_{q}^{s}
\end{array}\right)=\left(\begin{array}{cc}
S_{p p} & S_{q p} \\
S_{p q} & S_{q q}
\end{array}\right)\left(\begin{array}{c}
E_{p}^{i} \\
E_{q}^{i}
\end{array}\right)
$$

In this paper, we focused on two linear polarization representations and one circular polarization (Figure 2).

\section{A. Linear polarization}

When a linear polarization is selected, two main conventions are commonly used to express the scattering matrix : spherical convention - which is widespread in the microwave community [18] - and Bohren Huffman convention - commonly used in the light scattering community - [4].

\section{Spherical convention}

For a given transmitter/receiver pair, the scattering matrix can be constructed with a spherical convention ( $\mathrm{Sp}$ ), using the vectors $\left(\mathbf{e}_{\theta}^{i}, \mathbf{e}_{\phi}^{i}\right)$ as basis for the incident wave and the vectors $\left(\mathbf{e}_{\theta}^{s}, \mathbf{e}_{\phi}^{s}\right)$ as basis for the scattered wave (Figure 1). Therefore,
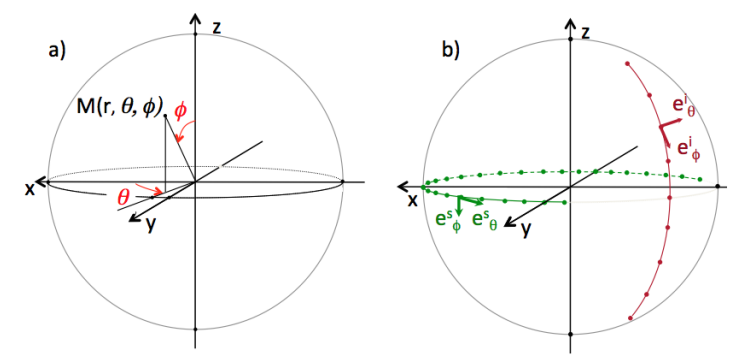

FIG. 1: Definition of the spherical convention: coordinate (a) and the polarization basis (b).

the element $S_{p, q}$ of the scattering matrix (with $p, q \in\{\phi, \theta\}$ ) corresponds to a scattered field measured along the vector $\mathbf{e}_{q}^{s}$ for an incident field $\mathbf{E}^{\mathbf{i}}$ polarized along the vector $\mathbf{e}_{p}^{i}$ (Figure 2(a)).
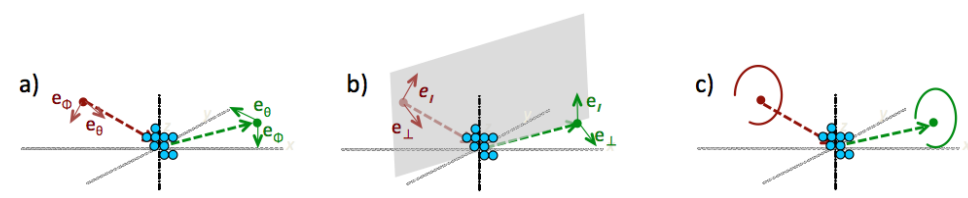

FIG. 2: Definition of the three basis: (a) Linear - Spherical convention, (b) Linear - Bohren and Huffman convention and (c) Circular.

\section{Bohren and Huffman convention}

In the Bohren and Huffman (BH) definition, the scattering elements are linked to the scattering plane, i.e, the plane containing both the incident wave vector $\mathbf{k}^{\mathbf{i}}$ and the scattered wave vector $\mathbf{k}^{\mathbf{s}}$ [19] (see Figure 2(b)). In the far-field region, the scattered field components $\left(E_{\|}^{s}\right.$ and $\left.E_{\perp}^{s}\right)$ are then linked to the incident ones $\left(E_{\|}^{i}\right.$ and $\left.E_{\perp}^{i}\right)$ by the scattering matrix. $E_{\|}^{i}$ (resp. $E_{\perp}^{i}$ ) corresponds to the parallel (resp. perpendicular) incident field component to the scattering plane and $E_{\|}^{s}$ (resp. $E_{\perp}^{s}$ ) corresponds to the parallel (resp. perpendicular) scattered field component to the scattering plane. The element $S_{p, q}$ of the scattering matrix (with $p, q \in\{\perp, \|\}$ ) corresponds to a scattered field measured along the $\mathbf{e}_{q}^{s}$ vector for an incident field $\mathbf{E}^{\mathbf{i}}$ polarized along the $\mathbf{e}_{p}^{i}$ vector (Figure 2(b)). The scattered field $E_{\perp}^{s}$ along $\mathbf{e}_{\perp}^{s}$ (resp. the scattered field $E_{\|}^{s}$ along $\mathbf{e}_{\|}^{s}$ ) is also called in polarization $S$ (resp. in polarization $P$ ).

The scattering matrix described in Bohren Huffman convention can be obtained by a simple basis change from the scattering matrix described in spherical convention and vice versa.

\section{B. Circular polarization}

The left-circular polarized incident (resp. scattered) wave $E_{l}^{i}$ (resp. $E_{l}^{s}$ ) and right-circular polarized wave $E_{r}^{i}\left(\right.$ resp. $\left.E_{r}^{s}\right)$ can be obtained from a linear combination of the linear polarized incident wave in the Bohren Huffman convention. In the

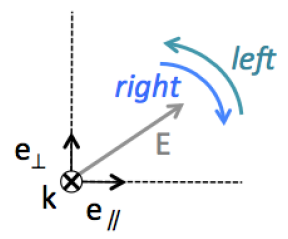

FIG. 3: Definition of the left and right circular polarization with the IEEE convention [20].

far field region and with the convention of the Figure 3 (outgoing wavevector for emission and incoming wavevector for reception) [20], [21], the scattering elements $S_{p, q}$ of the scattering matrix (with $p, q \in\{l, r\}$ ) can thus be expressed from the linear polarized elements in the Bohren Huffman conven- 
tion:

$$
\begin{aligned}
S_{r r} & =\frac{1}{2}\left[\left(S_{\|\|}-S_{\perp \perp}\right)-j\left(S_{\perp \|}+S_{\| \perp}\right)\right] \\
S_{r l} & =\frac{1}{2}\left[\left(S_{\|\|}+S_{\perp \perp}\right)-j\left(S_{\perp \|}-S_{\| \perp}\right)\right] \\
S_{l r} & =\frac{1}{2}\left[\left(S_{\|\|}+S_{\perp \perp}\right)+j\left(S_{\perp \|}-S_{\| \perp}\right)\right] \\
S_{l l} & =\frac{1}{2}\left[\left(S_{\|\|}-S_{\perp \perp}\right)+j\left(S_{\perp \|}+S_{\| \perp}\right)\right]
\end{aligned}
$$

\section{IMAGING PRINCIPLE}

The complex 3D permittivity map of the investigation zone is extracted from the fields measured in the receiving area by solving a non-linear inverse problem. In this work we started from an algorithm already implemented for the different cases of polarizations expressed in a spherical linear basis [22], [23]. For the current work, our algorithm has been adapted for the case where the scattering matrix is expressed in other basis. To do this, the cost functional, which estimates the difference between the measured scattering matrix element $S_{\mathcal{B}, p, q}^{m}$ and the simulated one $S_{\mathcal{B}, p, q}^{c}$ for a given permittivity map $\varepsilon_{r}^{\mathcal{B}, p, q(n)}$, both expressed in the basis $\mathcal{B}$, are written as follows

$\mathcal{F}_{\mathcal{B}, p, q}^{(n)}=\frac{1}{2 N_{s} N_{r}} \sum_{s=1}^{N_{s}} \sum_{r=1}^{N_{r}} \mid S_{\mathcal{B}, p, q}^{c}\left(\varepsilon_{r}^{\mathcal{B}, p, q(n)} ; \mathbf{r}_{s}, \mathbf{r}_{r}\right)-S_{\mathcal{B}, p, q}^{m}\left(\mathbf{r}_{s}, \mathbf{r}_{r}(\mathcal{A})\right.$

where $N_{s}$ (resp. $N_{r}$ ) is the number of source (resp. receiver) positions and $\varepsilon_{r}^{\mathcal{B}, p, q(n)}$ is the $\left(n^{\text {th }}\right) 3 \mathrm{D}$ reconstructed permittivity map with $\varepsilon_{r}^{\mathcal{B}, p, q}=\varepsilon_{r}^{\prime \mathcal{B}, p, q}+j \varepsilon_{r}^{\prime \prime} \mathcal{B}, p, q$. During the iterative process, this cost functional is minimized by an iterative procedure by modifying the permittivity map. Indeed, the permittivity of the voxels is updated through a gradient calculation:

$$
\varepsilon_{r}^{\mathcal{B}, p, q(n+1)}(\mathbf{r})=\varepsilon_{r}^{\mathcal{B}, p, q(n)}(\mathbf{r})+\alpha_{\mathcal{B}, p, q}^{(n)} d_{\mathcal{B}, p, q}^{(n)}
$$

with $\alpha_{\mathcal{B}, p, q}^{(n)}$ the optimal step, is determined by a classical line search to minimize the cost functional at each iteration. $d_{\mathcal{B}, p, q}^{(n)}$ is the descent direction derived from the gradients of the cost functional $\nabla_{\varepsilon_{r}} \mathcal{F}_{\mathcal{B}, p, q}{ }^{(n)}$ which are calculated with the adjoint problem [24]. At each step of the inversion procedure, the scattered field is calculated for the permittivity $\operatorname{map} \varepsilon_{r}^{\mathcal{B}, p, q(n)}$. The calculated scattered field is computed for the entire scattering matrix for the linear polarization in the spherical basis with a homemade forward problem based on a volume integral formulation and resolved with a biconjugated gradient stabilized FFT method [25] based on a 1D FFT to improve the calculation speed and to reduce the memory requirement [26] (see [27] for more details on this algorithm). The construction of the scattering matrix in the other basis is then deduced from the elements of the scattering matrix in the spherical basis. Initially, the permittivity of the voxels into the investigation domain is chosen to be very close to one (because of the constraint of positivity, a very low constant value is added to this value in order to avoid the case where the gradients disappear). The final permittivity map is obtained when the cost functional reaches a minimum.

Very weak a priori information is introduced on the permittivity map during the inversion procedure, i.e., we use only positivity constraints to ensure physically realistic values $\left(\varepsilon_{r}^{\prime \mathcal{B}, p, q} \geq 1\right.$ and $\left.\varepsilon_{r}^{\prime \prime} \mathcal{B}, p, q \geq 0\right)$ and, in order to concentrate only on the polarization effects, no regularization has been made.

\section{EXPERIMENTS}

The measurements, used for the $3 \mathrm{D}$ reconstructions, were made in the anechoic chamber of the CCRM (Centre Commun de Ressources en Microonde) (Figure 4 (left)). A spherical exprimental setup is included in this anechoic chamber, a sketch of its positioning devices is presented in Figure 4 (right)). Thanks to the different possible azimuthal and elevation displacements of the antennas in the setup, it is possible to measure the scattered field strength over a large part of a sphere surrounding the target. The target under test is placed at the center of a sphere of around $1.7 \mathrm{~m}$ diameter on a vertical expanded polystyrene mast considered transparent to microwaves. Two horn antennas, which transmit alternately, are placed on a circular vertical arch and each can have a total angular range of $180^{\circ}$. The two transmitting antennas are in $\mathbf{e}_{\phi}$ and $\mathbf{e}_{\theta}$ polarizations respectively. The polarization of each antenna with respect to the spherical setup is selected by the antenna rotation as they are linearly polarized. A receiving horn antenna can also move circularly around the target in the azimuthal plane to capture the scattered signal, with a total angular range of $260^{\circ}$. The target can make a full rotation $\left(360^{\circ}\right)$ around the vertical axis. More details on this experimental setup can be found in [28], [29]. In this study, in order to acquire the measured scattered fields corresponding to the four cases of co-polarization and cross polarization, two measurements are made. A first measurement with the receiving antenna polarization fixed at $\mathbf{e}_{\phi}$ allows to achieve the $\phi \phi$ and $\theta \phi$ measurement cases, and a second measurement with the receiving antenna polarization fixed at $\mathbf{e}_{\theta}$ allows to achieve the $\phi \theta$ and $\theta \theta$ measurement cases. With the experimental setup, the signal is measured in both magnitude and phase which allows to obtain the complex value of the electric field. The scattered electric field $E_{p, q}^{s}$ cannot be obtained in a unique step, but it is rather obtained from the complex subtraction of two different fields: the measured electric field with the target under test is at its place in the chamber (total field) and measured electric field with no target (incident field). After obtaining the scattered field, a drift-correction procedure is applied [30] to remove the drift errors that could happen due to the time delay between the total and incident field. At the end, the drift corrected scattered field is calibrated, using a measurement of a reference target, to refer the incident field to a magnitude equal to one and a null phase at the target's center. 

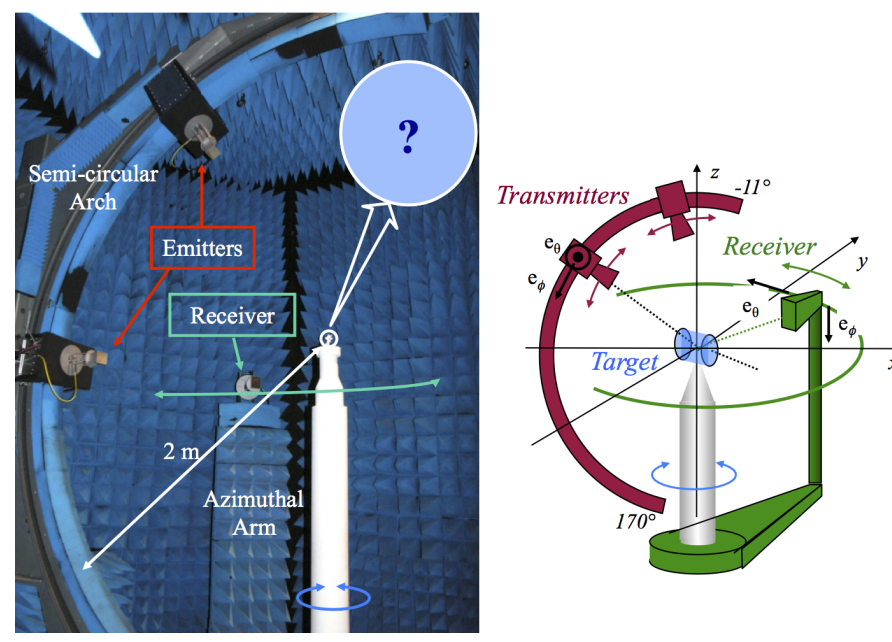

FIG. 4: Experimental setup

\section{IMAGING RESULTS}

\section{A. Targets}

We consider two targets, i.e., the first is a canonical object with a relatively low permittivity value. It is a small cube with permittivity equal to $\varepsilon_{r}=1.45$. Its side is equal to $20 \mathrm{~mm}$ $(1.2 \lambda$ at $18 \mathrm{GHz})$. The results for the first target may, in some cases, be interpreted with approximations on the induced current (see subsection VI B). The second object has a more complicated shape, i.e., two dimensions are very small compared to the wavelength ( $<\frac{\lambda}{3}$ at the work frequency). Unlike the previous object, with this second object, the multiple scattering effect plays an important role, that cannot be neglected in the interpretation. This second target is a circular helix, made by the Centre de Transfert de Technologies du Mans through additive manufacturing (Figure 5). It can be included in a sphere of diameter $70 \mathrm{~mm}(4.2 \lambda$ at $18 \mathrm{GHz})$. Its length is $45 \mathrm{~mm}$ and its diameter $40 \mathrm{~mm}$. The wire diameter is $5 \mathrm{~mm}$ $\left(<\frac{\lambda}{3}\right.$ at $18 \mathrm{GHz}$ ). The aspect ratio of the equivalent cylinder, defined as the ratio between radius and length, is equal to 0.135 . This target was built in acrylate and its permittivity was measured with a method based on the bistatic far-field scattering patterns of a sphere made with the same material [31] and was found equal to $\varepsilon_{r}=(2.97 \pm 0.02)+j(0.085 \pm 0.001)$ at $18 \mathrm{GHz}$ [32].

\section{B. Reconstructed 3D permittivity maps}

This paragraph is devoted to the presentation of the 3D reconstructed permittivity maps. All the inversions are performed at $18 \mathrm{GHz}$, with 99 source positions $\left(\phi_{s} \in\left[30^{\circ}\right.\right.$ : $\left.\left.10^{\circ}: 130^{\circ}\right], \theta_{s} \in\left[110^{\circ}: 40^{\circ}: 430^{\circ}\right]\right)$ and 53 receivers $\left(\phi_{r}=90^{\circ}, \theta_{r} \in\left[50^{\circ}+\theta_{s}: 5^{\circ}: 310^{\circ}+\theta_{s}\right]\right)$. These angles refer to the spherical coordinates of our experimental setup (Figure 4). The investigation zone is a cubic zone with a side of $30 \mathrm{~mm}\left(6 \lambda^{3}\right.$ at $\left.18 \mathrm{GHz}\right)$ for the cube and a parallelepipedic area $(49 \times 95 \times 82) \mathrm{mm}^{3}\left(83 \lambda^{3}\right.$ at $\left.18 \mathrm{GHz}\right)$ for the circular helix.

To compare the various reconstructions in a quantitative way, some criteria are introduced to compare the $N$ voxels of the reconstructed map with the ones of the theoretical map. It can be noticed that misalignment errors in the measurements can introduce a bias in the value of these criteria (but misalignment errors are low, as shown in [18]). Five criteria are considered, plus one $\mathcal{C}_{\mathcal{B}}$ that mixes the different effects. The first one is the classical quadratic norm $C_{\mathcal{B}}^{L 2}$. As we shall see, this criterion is often "good" even "very good" and is too weakly sensitive to differences between maps. Two other criteria are also used, $C_{\mathcal{B} \text {,with }}^{L 2}$ and $C_{\mathcal{B} \text {,empty }}^{L 2}$, that are also quadratic norms, but calculated by considering only the voxels with material and only the empty voxels (taking as reference the theoretical map). The fourth criterion is a crosscorrelation between the reconstructed and theoretical $\varepsilon_{r}-1$ maps, obtained for the correct geometrical offset $\mathbf{d}^{*}$ between the two maps. $C_{\mathcal{B}}^{c}$ is the correlation between the two maps.

$$
\begin{gathered}
C_{\varepsilon_{r}-1, \mathcal{B}}^{c c}\left(\mathbf{d}^{*}\right)=\frac{\sum_{N}\left(\varepsilon_{r}^{\text {true }}\left(\mathbf{r}+\mathbf{d}^{*}\right)-1\right) \overline{\left(\varepsilon_{r}^{\text {reco }}\left(\mathbf{d}^{*}\right)-1\right)}}{\sum_{N}\left(\varepsilon_{r}^{\text {true }}(\mathbf{r}+\mathbf{0})-1\right) \overline{\left(\varepsilon_{r}^{\text {true }}(\mathbf{0})-1\right)}}(6) \\
C_{\mathcal{B}}^{c}=\frac{\sum_{N}\left(\varepsilon_{r, \mathcal{B}}^{\text {reco }}(\mathbf{r})-\overline{\varepsilon_{r, \mathcal{B}}^{\text {reco }}}\right)\left(\varepsilon_{r}^{\text {true }}(\mathbf{r})-\overline{\varepsilon_{r}^{\text {true }}}\right)}{\sqrt{\sum_{N}\left(\varepsilon_{r, \mathcal{B}}^{\text {reco }}(\mathbf{r})-\overline{\varepsilon_{r, \mathcal{B}}^{\text {reco }}}\right)^{2} \sum_{N}\left(\varepsilon_{r}^{\text {true }}(\mathbf{r})-\overline{\varepsilon_{r}^{\text {true }}}\right)^{2}}}(7) \\
\left.\mathcal{C}_{\mathcal{B}}=\left(1-C_{\mathcal{B}}^{L 2}\right) \times C_{\varepsilon_{r}-1, \mathcal{B}}^{c c} \times C C_{\mathcal{B}}\right)
\end{gathered}
$$

It can be noticed that for these criteria $\left(1-C_{\mathcal{B}}^{L 2}, 1-C_{\mathcal{B}, \text { with }}^{L 2}\right.$, $\left.1-C_{\mathcal{B}, \text { empty }}^{L 2}, C_{\varepsilon_{r}-1, \mathcal{B}}^{c c}, C_{\mathcal{B}}^{c}, C_{\mathcal{B}}\right)$ the best value is 1 .

\section{Cube reconstructions}

For the cube, we perform the inversion from calculated scattered fields. These scattered fields were calculated with the same home-made forward software used for the inversion procedure, based on a volume integral formulation [27]. To avoid the "inverse crime", the discretization of the target zone is not similar in this forward problem and in the inversion procedure. As it is explained in the section VI, we present in this section only the co-polarization and the well-adapted polarization elements. In figures 6,7 and 8 , the permittivity maps in the three planes $x=0 \mathrm{~mm}, y=0 \mathrm{~mm}$ and $z=0 \mathrm{~mm}$ are plotted in the various polarization cases. The criteria values are summarized in table I. 

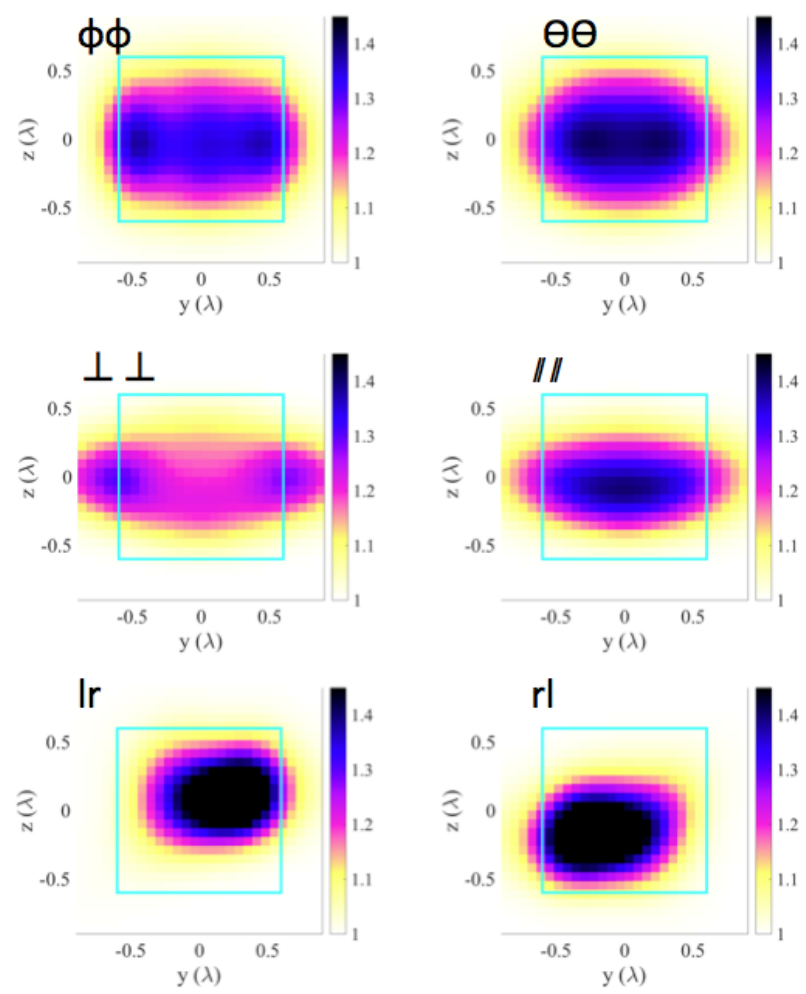

FIG. 6: 2D cross section in the $(y 0 z)$ plane at $x=0 \mathrm{~mm}$ of the reconstructed maps in the principal polarization cases from calculated scattered fields for the cube case. The edges of the target are superimposed in cyan.
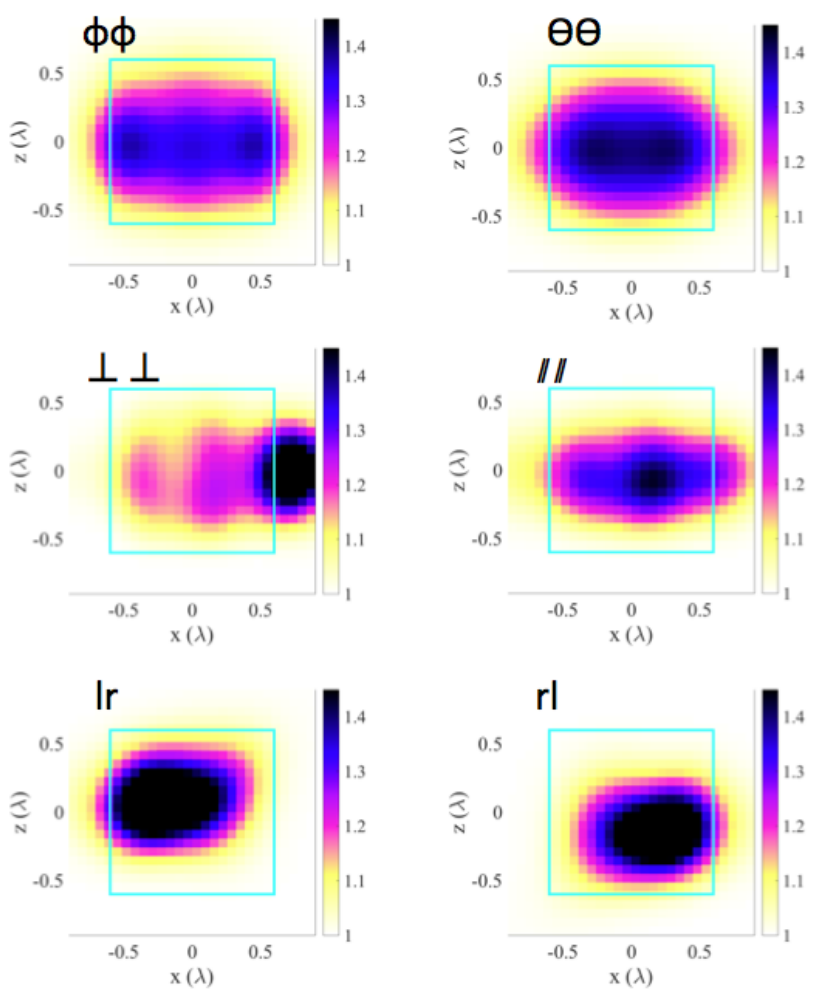

FIG. 7: $2 \mathrm{D}$ cross section in the $(x 0 z)$ plane at $y=0 \mathrm{~mm}$ of the reconstructed maps in the principal polarization cases from calculated scattered fields for the cube case. The edges of the target are superimposed in cyan.

\begin{tabular}{|c|c|c|c|c|c|c|c|c|c|c|c|c|c|}
\hline & & & & & & & Pola. & $1-C_{\mathcal{B}}^{L 2}$ & $1-C_{\mathcal{B}, \text { empty }}^{L 2}$ & $1-C_{\mathcal{B}, \text { with }}^{L 2}$ & $C^{c}$ & $C_{\varepsilon_{r}-1, \mathcal{B}}^{c c}$ & $\mathcal{C}_{\mathcal{B}}$ \\
\hline Pola. & $1-C_{\mathcal{B}}^{L 2}$ & $1-C_{\mathcal{B}, \text { empty }}^{L 2}$ & $1-C_{\mathcal{B}, \text { with }}^{L 2}$ & $C^{c}$ & $C_{\varepsilon_{r}-1, \mathcal{B}}^{c c}$ & $\overline{\mathcal{C}_{\mathcal{B}}}$ & $\phi \phi$ & 0.917 & 0.993 & 0.924 & 0.522 & 0.159 & 0.076 \\
\hline$\phi \phi$ & 0.973 & 0.999 & 0.974 & 0.837 & 0.415 & 0.338 & $\theta \theta$ & 0.921 & 0.994 & 0.928 & 0.542 & 0.159 & 0.079 \\
\hline$\theta \theta$ & 0.971 & 0.999 & 0.973 & 0.814 & 0.402 & 0.318 & $\perp \perp$ & 0.895 & 0.986 & 0.909 & 0.194 & 0.103 & 0.018 \\
\hline$\perp \perp$ & 0.952 & 0.992 & 0.960 & 0.688 & 0.257 & 0.168 & \|\| & 0.901 & 0.987 & 0.915 & 0.266 & 0.120 & 0.102 \\
\hline\|\| & 0.961 & 0.998 & 0.962 & 0.796 & 0.280 & 0.215 & $l r$ & 0.923 & 0.992 & 0.931 & 0.517 & 0.213 & 0.102 \\
\hline$l r$ & 0.960 & 0.999 & 0.961 & 0.656 & 0.354 & 0.223 & $r l$ & 0.923 & 0.992 & 0.931 & 0.528 & 0.205 & 0.100 \\
\hline$r l$ & 0.960 & $\approx 1$ & 0.961 & 0.656 & 0.354 & 0.223 & & & & & & & \\
\hline
\end{tabular}

TABLE I: Comparison of the different reconstruction cases from the calculated scattered field. The target is the cube at the frequency of $18 \mathrm{GHz}$. It can be noticed that these criteria are better when there are 1 .

\section{Circular helix reconstructions}

For this target, the inversion was done both from measured scattered fields and from calculated scattered fields. The criteria values, summarized in the tables II and III, were calculated for the reconstructions from the two cases. We present only the reconstructed maps obtained from the measurements (see Figure 9 for the 3D views and Figures 10, 11 and 12 for the permittivity maps).
TABLE II: Comparison of the different reconstruction cases from calculated scattered field. The target is the circular helix at the frequency of $18 \mathrm{GHz}$.

\section{Comparison of the different polarization cases}

As it can be seen in Tables II and III, the maps reconstructed from measurements for the circular helix are close to those obtained from calculations in the different polarization cases. However, the maps reconstructed from experimental fields have higher discrepancy (in particular the $\theta \theta$ case). This is logical, especially since no information on the experimental noise is taken into account in the inversion procedure, i.e., no regularization is carried out in this inversion concerning this noise [22].

In tables I, II and III, we can see that the quadratic standard norm calculated on all voxels $\left(1-C_{\mathcal{B}}^{L 2}\right)$ is very good (val- 

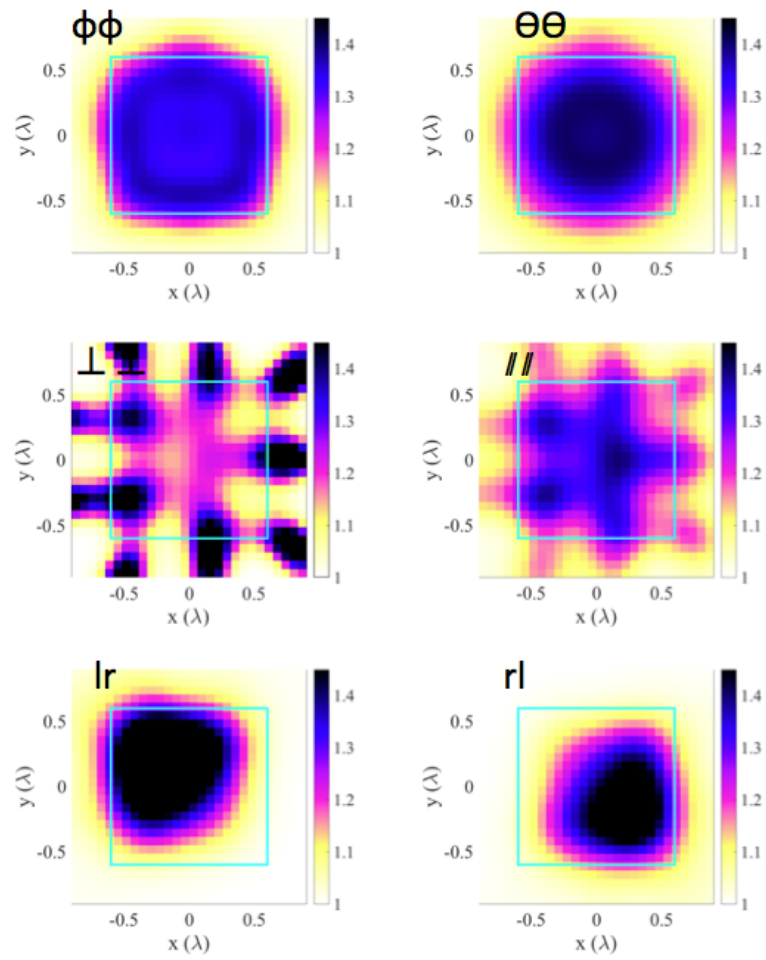

FIG. 8: 2D cross section in the $(x 0 y)$ plane at $z=0 \mathrm{~mm}$ of the reconstructed maps in the principal polarization cases from calculated scattered fields for the cube case. The edges of the target are superimposed in cyan.
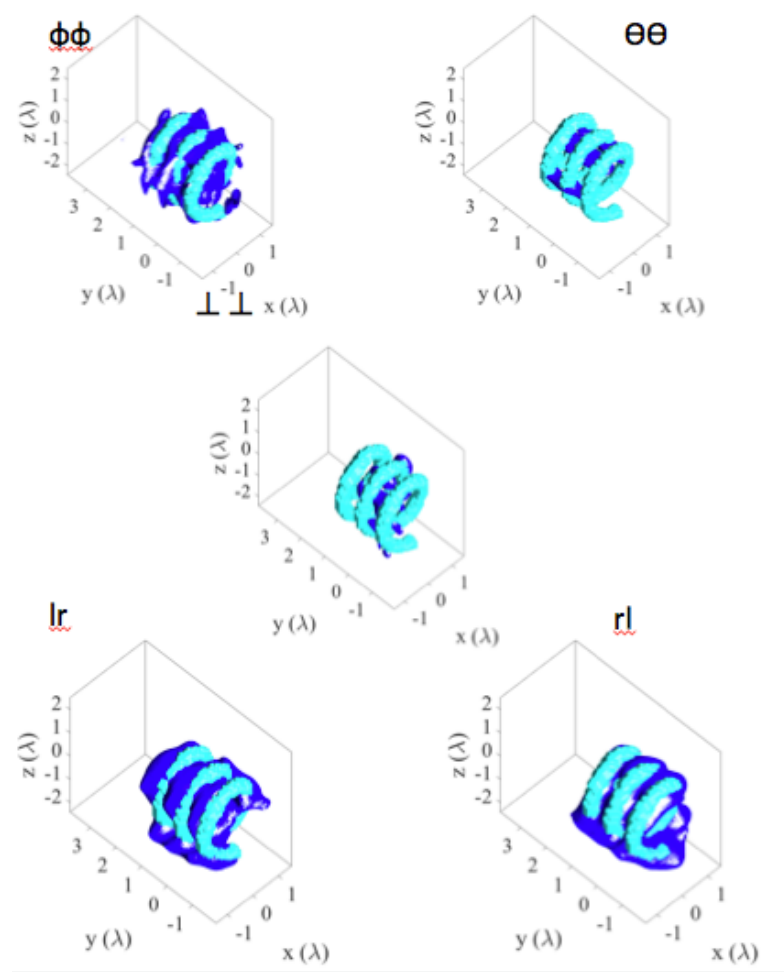

FIG. 9: 3D view of the reconstructed maps in the different polarization cases from measured scattered fields for the circular helix (the threshold is chosen at 1.1). The real object is superimposed in cyan.

\begin{tabular}{|c|c|c|c|c|c|c|}
\hline Pola. & $1-C_{\mathcal{B}}^{L 2}$ & $1-C_{\mathcal{B}, \text { empty }}^{L 2}$ & $1-C_{\mathcal{B}, \text { with }}^{L 2}$ & $C_{\mathcal{B}}^{c}$ & $C_{\varepsilon_{r}-1, \mathcal{B}}^{c c}$ & ion of this criterion. Considering $C_{\mathcal{B} \text {,with }}^{L 2}$, the vo \\
\hline$\phi \phi$ & 0.915 & 0.992 & 0.923 & 0.246 & 0.143 & 0.032 \\
\hline$\theta \theta$ & 0.915 & $\approx 1$ & 0.915 & 0.297 & 0.031 & $0.008 \phi \phi, \theta \theta$ for th \\
\hline$\perp \perp$ & 912 & $\approx 1$ & 913 & 127 & 17 & $0.002 p \phi, \theta \theta, l r$ \\
\hline\|\| & 0.912 & $\approx 1$ & 0.912 & 0.194 & 0.002 & $\approx 0$ criterion and are better than the two $\mathrm{p}$ \\
\hline$l r$ & 0.927 & 0.990 & 0.936 & 0.442 & 0.227 & 0.093 asis $(\perp \perp$ and \\
\hline$r l$ & 0.915 & 0.989 & 0.926 & 0.282 & 0.220 & $0.05 \pi$ he cross correlation $C_{\varepsilon_{r}}^{c c}$ \\
\hline
\end{tabular}

TABLE III: Comparison of the different reconstruction cases from the measured scattered field. The target is the circular helix at the frequency of $18 \mathrm{GHz}$.

ues close to one) in all the polarization cases for the two objects. It is important to note that this criterion is the most common. Nevertheless, it gives only a global information and is not very discriminating. Indeed, it mainly provides information on empty cells, especially when they are in majority. Then, to compare the results in details, other criteria have been used. One solution is to separate the cells with material (according to the theoretical map) from the empty cells and to calculate the quadratic norm on these two sets $\left(1-C_{\mathcal{B}, \text { empty }}^{L 2}\right.$, $\left.1-C_{\mathcal{B}, \text { with }}^{L 2}\right)$. Considering that, $1-C_{\mathcal{B}, \text { empty }}^{L 2}$, empty voxels are well reconstructed for the two objects in all polarization cases except in the $\perp \perp$ case. Indeed, in this polarization case, there are artefacts on the reconstructed maps, which results in reconstruction (voxels that should be empty but are not on the reconstructed map) or (ii) if a part of the target is not reconstructed or (iii) if the object's permittivity value is not well recovered. $C_{\varepsilon_{r}-1, \mathcal{B}}^{c c}$ is very low for the cases $\perp \perp$ and \|\| for the two targets. For these two polarization cases, this is because many parts of the object are not reconstructed (see Figures 9 12) and because, specifically for the $\perp \perp$ case, there are many artifacts (see Figures 6 -8). For the circular helix, in $\phi \phi$ and $\theta \theta$ cases, this criterion has an average value. For $\phi \phi$, there are artifacts on the reconstructed map and for $\theta \theta$, the value of the reconstructed permittivity is too low. The two best cases are $l r$ and $r l$, where the reconstituted value of the permittivity is well recovered and there are few artifacts. For the cube case, the best values of this criterion are for the $\phi \phi$ and $\theta \theta$ cases, the ones where the entire shape of the object is better reconstructed. Finally, the $C^{c}$ correlation, that takes also into account the empty voxels, goes in the same direction, i.e., the best reconstruction cases are the ones in circular polarization for the circular helix and $\phi \phi$ and $\theta \theta$ for the cube. The global criterion $\mathcal{C}_{\mathcal{B}}$, which is a simple multiplication of the three cri- 

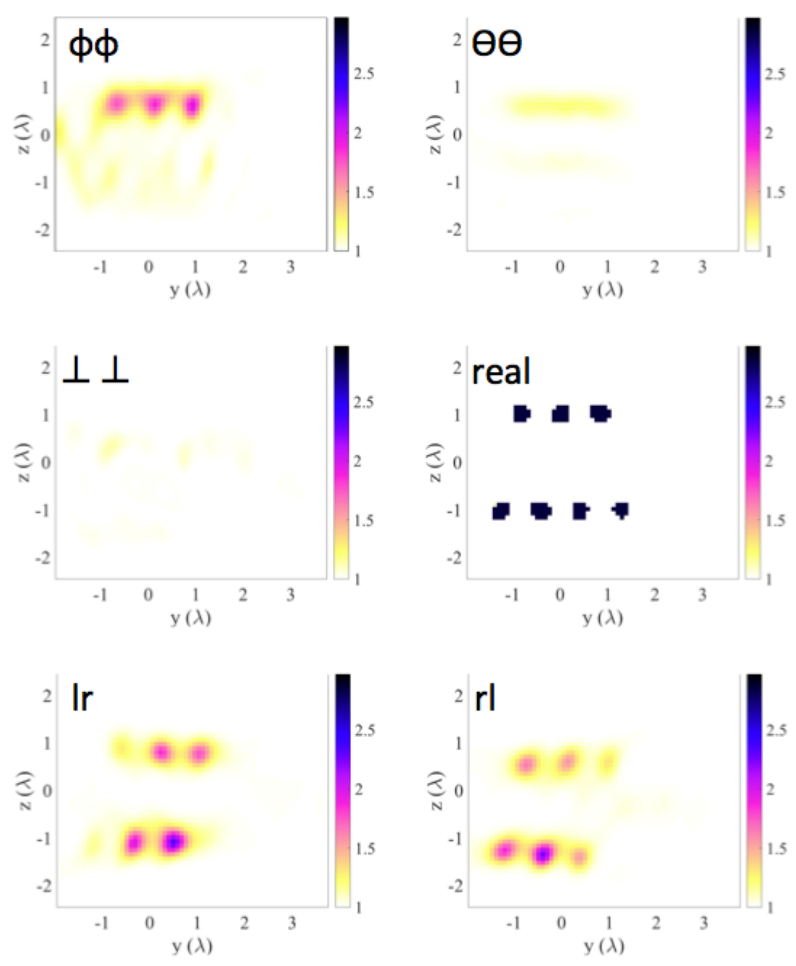

FIG. 10: 2D cross section in the $(x 0 y)$ plane at $z=0 \mathrm{~mm}$ of the reconstructed maps in the principal polarization cases from measured scattered fields for the circular helix.

teria, confirms that.

\section{ANALYSIS OF THE POLARIZATION EFFECTS}

This section is devoted to the analysis of differences in reconstructions from the various elements of the scattering matrix.

\section{A. Singular Value Decomposition}

To analyze the information content in each scattering matrix element $\left[\mathbf{S}_{\mathcal{B}, \mathbf{p q}}\right]$ of polarization $(p q)$ in the basis $\mathcal{B}$, a Singular Value Decomposition of this multistatic element has been made.

$$
\left[\mathbf{S}_{\mathcal{B}, p q}\right]=\left[\mathbf{U}_{\mathcal{B}, p q}\right]\left[\boldsymbol{\Lambda}_{\mathcal{B}, p q}\right]\left[\mathbf{V}_{\mathcal{B}, p q}\right]
$$

with $\left[\boldsymbol{\Lambda}_{\mathcal{B}, p q}\right]$, the matrix that contains the singular values, $\left[\mathbf{U}_{\mathcal{B}, p q}\right]$ and $\left[\mathbf{V}_{\mathcal{B}, p q}\right]$, the two matrix that contain the associated bases singular vectors for $(p, q) \in[\phi, \theta, \perp, \|, l, r]$. All these quantities expressed in the basis $\mathcal{B}$ for a scattered electric field measured along $\mathbf{e}_{\mathbf{q}}^{\mathbf{s}}$ and for an incident electric field polarized along $\mathbf{e}_{\mathbf{p}}^{\mathbf{i}}$.

Figures 13 and 14 show, for the two objects, the sum of the spectrum in each polarization case considering the spherical
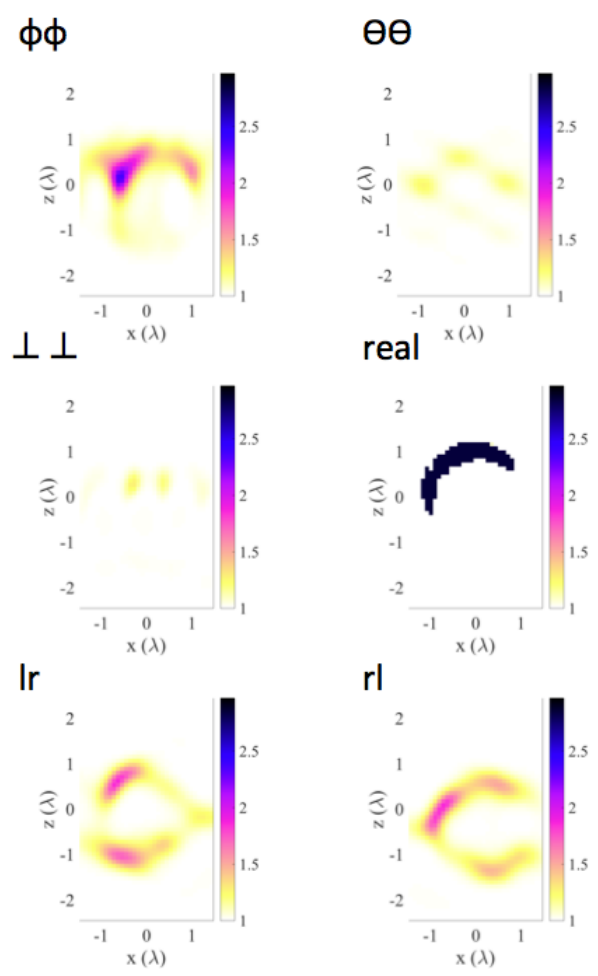

FIG. 11: 2D cross section in the $(x 0 z)$ plane at $y=0 \mathrm{~mm}$ of the reconstructed maps in the principal polarization cases from measured scattered fields for the circular helix.

basis, the $\mathrm{BH}$ basis and the circular basis. The cases that contain most information are the co-polarization elements $\left(S_{\phi \phi}\right.$, $\left.S_{\theta \theta}, S_{\perp \perp}, S_{\|\|}\right)$and the well-adapted circular polarization elements $\left(S_{l r}, S_{r l}\right)$. To take a closer look at the spectra, the items with most information are detailed in Figures 15 and 16. The size of the signal space for all polarization cases appears to be the same. This is also in agreement with the Efficient Bandwidth approach [33]. Indeed, with such an approach, for a target of this size, illuminated at this frequency, the width of this spectrum should be, given our limited circular arc, equal to 16 for the cube case and 27 for the circular helix case.

As might be expected, the co-polarized elements $\left(S_{\phi \phi}, S_{\theta \theta}\right.$, $\left.S_{\theta \theta}, S_{\perp \perp}, S_{\|\|}\right)$and the well-adapted circular polarized elements $\left(S_{r l}, S_{l r}\right)$ contain more information than the crosspolarized elements $\left(S_{\phi \theta}, S_{\theta \phi}, S_{\perp \|}, S_{\| \perp}\right)$ or than the notadapted circular polarized elements $\left(S_{r r}, S_{l l}\right)$. Differences between the co-polarized/well-adapted circular polarized elements $\left(S_{\phi \phi}, S_{\theta \theta}, S_{\theta \theta}, S_{\perp \perp}, S_{\|\|}, S_{r l}, S_{l r}\right)$ are small and appear to be relative to the target (below, an analysis for the two targets under studied). This multistatic matrix analysis does not allow to discriminate between these elements. In particular, it does not allow to choose one of the elements rather than another.

For the cube case, the sum of the singular values of the elements $S_{l r}, S_{r l}, S_{\phi \phi}$ and $S_{\perp \perp}$ is a bit higher than the elements $S_{\theta \theta}$ and $S_{\|\|}$) (Figure 13). These elements correspond also to the ones having the higher values in the signal space (Figure 15). Within these elements, the differences are too small to 


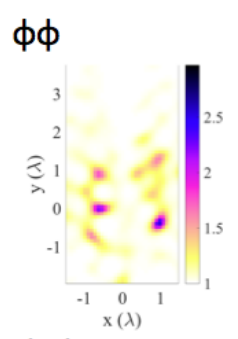

$\perp \perp$

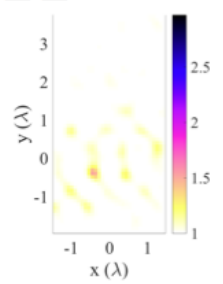

Ir

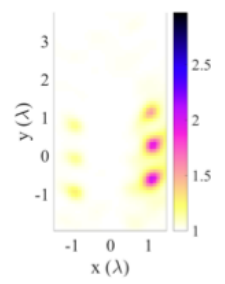

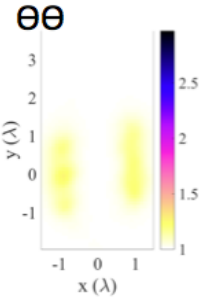

real

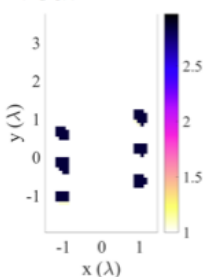

$\mathrm{rl}$

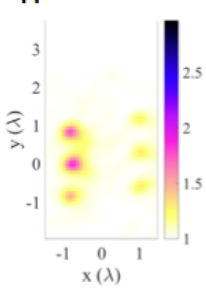

FIG. 12: 2D cross section in the $(y 0 z)$ plane at $x=0 \mathrm{~mm}$ of the reconstructed maps in the principal polarization cases from measured scattered fields for the circular helix.
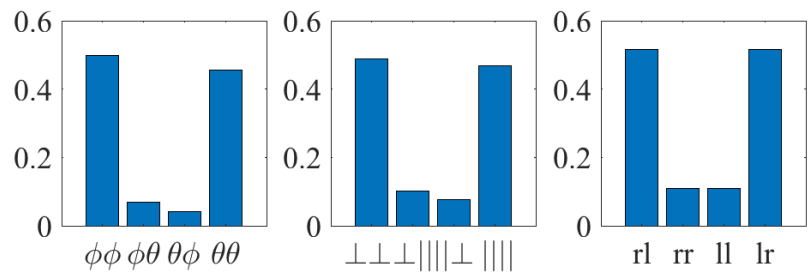

FIG. 13: Sum of all the singular values for the Cube (left: in spherical basis, middle: in BH basis and right: in circular basis) $\left(\sum_{n=1}^{N_{r}} \lambda_{\mathcal{B} p q}^{(n)}\right)$.
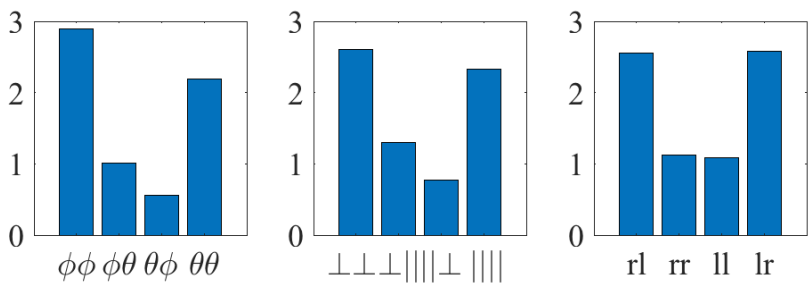

FIG. 14: Sum of all the singular values for the circular helix (left: in spherical basis, middle: in BH basis and right: in circular basis) $\left(\sum_{n=1}^{N_{r}} \lambda_{\mathcal{B} p q}^{(n)}\right)$.

discriminate.

For the circular helix case, as it can be seen, all the singular
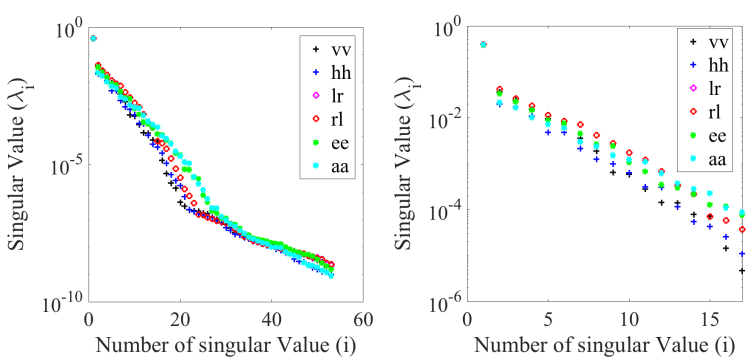

FIG. 15: Spectra of singular values $\lambda_{\mathcal{B}, p q}^{(n)}$ for the Cube: full spectra (left) and spectra corresponding to the signal space (right).
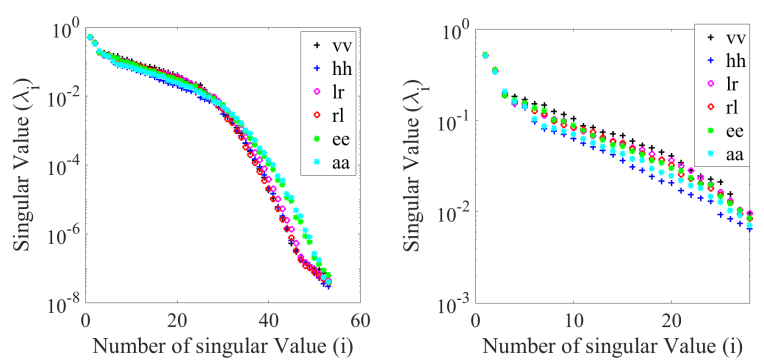

FIG. 16: Spectra of singular values $\lambda_{\mathcal{B}, p q}^{(n)}$ for the circular helix: full spectra (left) and spectra corresponding to the signal space (right).

values of the $S_{\phi \phi}$ elements are higher than the those of the other elements in the signal space (only the first three singular values are almost identical for all the polarization cases) (Figure 16). Then, the spectra of the elements $S_{\perp \perp}, S_{l r}, S_{r l}$ are almost similar and are higher than the spectra of the elements $S_{\|\| \|}$and $S_{\theta \theta}$.

\section{B. Polarization vector analysis}

In this section, we analyze, in the case of linear polarization, the filter effect created by the scalar product between the transmitter and the receiver polarization directions. It should be noticed that in the case of the circular base, this effect does not exist. As it is explained in the following, we restrict our analysis to the cases where, multiple scattering effects are weak as a first approximation.

For a source position at $\mathbf{r}_{\mathbf{s}}$ and a receiver position at $\mathbf{r}_{\mathbf{r}}$, the scattered field can be written as

$$
\mathbf{E}^{\mathbf{S}}\left(\mathbf{r}_{\mathbf{s}}, \mathbf{r}_{\mathbf{r}}\right)=\int_{\Omega} \mathbf{G}\left(\mathbf{r}_{\mathbf{r}}, \mathbf{r}^{\prime}\right) \chi\left(\mathbf{r}^{\prime}\right) \mathbf{E}\left(\mathbf{r}^{\prime}\right) d \mathbf{r}^{\prime}
$$

with $\mathbf{G}$ the dyadic Green function, $\chi\left(\mathbf{r}^{\prime}\right)=k^{2}\left(\mathbf{r}^{\prime}\right)-k_{o}^{2}$, the contrast term ( $k_{o}$ being the wavenumber in the free space) and $\mathbf{E}$, the electric field vector. The scattered field measured by the receiver, polarized along $\mathbf{e}_{\mathcal{B}, q}^{s}$, is:

$$
\mathbf{E}^{\mathbf{s}}\left(\mathbf{r}_{\mathbf{s}}, \mathbf{r}_{\mathbf{r}}\right) \cdot \mathbf{e}_{\mathcal{B}, q}^{s}=\left(\int_{\Omega} \mathbf{G}\left(\mathbf{r}_{\mathbf{r}}, \mathbf{r}^{\prime}\right) \chi\left(\mathbf{r}^{\prime}\right) \mathbf{E}\left(\mathbf{r}^{\prime}\right) d \mathbf{r}^{\prime}\right) \cdot \mathbf{e}_{\mathcal{B}, q}^{s}(11)
$$




\begin{tabular}{|c|c|c|c|c||c|c|c|c|}
\hline $\mathcal{B}$ & $S p$ & $S p$ & $S p$ & $S p$ & $B H$ & $B H$ & $B H$ & $B H$ \\
\hline$p, q$ & $\phi \phi$ & $\phi \theta$ & $\theta \phi$ & $\theta \theta$ & $\perp \perp$ & $\perp \|$ & $\| \perp$ & \|\| \\
\hline$Q_{\mathcal{B}}$ & 0.84 & 0.30 & 0 & 0.55 & 1 & 0 & 0 & 0.46 \\
\hline
\end{tabular}

TABLE IV: Values of the quantity $Q_{\mathcal{B}}$ for different polarization cases.

The induced current vector in the target zone $\Omega$, for a permittivity map $\varepsilon_{r}(\mathbf{r})$, obtained for an incident field vector $\mathbf{E}^{\mathbf{i}}{ }_{\mathcal{B}, p}=E^{i} \mathbf{e}_{\mathcal{B}, p}^{i}$ is:

$$
\forall \mathbf{r} \in \Omega, \mathbf{J}_{\mathcal{B}, p}(\mathbf{r})=\chi(\mathbf{r}) \mathbf{E}_{\mathcal{B}, p}(\mathbf{r})
$$

This analysis is restricted to scattering with small multiple scattering effects and we assume that the induced current $\mathbf{J}_{\mathcal{B}, p}=\chi \mathbf{E}_{\mathcal{B}, p}$ is collinear to the incident field

$$
\mathbf{E}^{\mathbf{s}}\left(\mathbf{r}_{\mathbf{s}}, \mathbf{r}_{\mathbf{r}}\right) \cdot \mathbf{e}_{\mathcal{B}, q}^{s} \approx\left[\int_{\Omega} \mathbf{G}\left(\mathbf{r}_{\mathbf{r}}, \mathbf{r}^{\prime}\right) J_{\mathcal{B}, p}\left(\mathbf{r}^{\prime}\right) d \mathbf{r}^{\prime} \mathbf{e}_{\mathcal{B}, p}^{i}\right] \cdot \mathbf{e}_{\mathcal{B}, q}^{s}(13)
$$

In the far field conditions, i.e. when each receiver position $\mathbf{r}_{\mathbf{r}}$ in the receiver zone $\Gamma$, satisfies the following conditions:

$$
\forall \mathbf{r}_{\mathbf{r}} \in \Gamma, \forall \mathbf{r}^{\prime} \in \Omega, \quad k_{o} r_{r} \gg 1, \quad r_{r} \gg r^{\prime} \text { et } \frac{k_{o} r^{\prime}}{2 r_{r}} \gg(114)
$$

G can be approximated by [4]:

$$
\mathbf{G}\left(\mathbf{r}_{\mathbf{r}}, \mathbf{r}^{\prime}\right) \approx \frac{e^{j k_{o} r_{r}}}{4 \pi r_{r}} e^{-j k_{o} \mathbf{e}_{\mathcal{B}, \mathbf{q}}^{\mathbf{s}} \cdot \mathbf{r}^{\prime}}\left[\mathbf{e}_{\theta} \otimes \mathbf{e}_{\theta}+\mathbf{e}_{\phi} \otimes \mathbf{e}_{\phi}\right]
$$

The the scattered field measured by the receiver can thus be written as follows:

$$
\begin{array}{r}
\mathbf{E}^{\mathbf{s}}\left(\mathbf{r}_{\mathbf{s}}, \mathbf{r}_{\mathbf{r}}\right) \cdot \mathbf{e}_{\mathcal{B}, q}^{s} \approx\left(\int_{\Omega} J_{\mathcal{B}, p}\left(\mathbf{r}^{\prime}\right) e^{-j k_{o} \mathbf{e}_{\mathcal{B}, q}^{s} \cdot \mathbf{r}^{\prime}} d r^{\prime}\right) \\
g\left(r_{r}\right)\left[\mathbf{e}_{\theta} \otimes \mathbf{e}_{\theta}+\mathbf{e}_{\phi} \otimes \mathbf{e}_{\phi}\right] \mathbf{e}_{\mathcal{B}, p}^{i} \cdot \mathbf{e}_{\mathcal{B}, q}^{s}
\end{array}
$$

with $g\left(r_{r}\right)=\frac{e^{j k_{o} r_{r}}}{4 \pi r_{r}}$.

Under the previous assumptions, we were interested in the part of the field which is not filtered by the scalar product between the polarization of the transmitting antenna and the polarization of the receiving antenna. Note that this quantity does not depend on the object, it only depends on the geometry of the configuration, i.e., it changes depending on the position of the source and receiver. This provides information on the main directions examined in the target. The quantity $Q_{\mathcal{B}}$ is constructed as follows:

$$
Q_{\mathcal{B}}=\frac{1}{N_{s} N_{r}} \sum_{N_{s}} \sum_{N_{r}} q_{\mathcal{B}}\left(\phi_{s}, \theta_{s}, \theta_{s}-\theta_{r}\right)
$$

with $q_{\mathcal{B}}\left(\phi_{s}, \theta_{s}, \theta_{s}-\theta_{r}\right)=\left|\left[\left(\mathbf{e}_{\theta} \otimes \mathbf{e}_{\theta}+\mathbf{e}_{\phi} \otimes \mathbf{e}_{\phi}\right) \mathbf{e}_{\mathcal{B}, p}^{i}\right] \cdot \mathbf{e}_{\mathcal{B}, q}^{s}\right|$ and its are provided in Table IV.

According to Table IV, the $\perp \perp$ seems to be the best case: $Q_{\mathcal{B}}$ is equal to one for this polarization case and none of the measurement points is filtered in this polarization. Then, the $\phi \phi$ case is an interesting case because $Q_{\mathcal{B}}$ is high and all

\begin{tabular}{|c||c|c||c|c|}
\hline $\mathcal{B}$ & $S p$ & $S p$ & $B H$ & $B H$ \\
\hline$p$ & $\phi$ & $\theta$ & $\perp$ & $\|$ \\
\hline $\mathcal{A}_{\mathcal{B}, p}$ & 0.985 & 0.985 & 0.057 & 0.056 \\
\hline
\end{tabular}

TABLE V: Values of the quantity $\mathcal{A}_{\mathcal{B}, p}$ for the different illuminations of the two linear basis in the cube case.

measurements contribute. The part of the measurement that has the lowest weight corresponds to the smallest values of the angle $\phi_{s} . \theta \theta$ and \|\| correspond to low values of $Q_{\mathcal{B}}$ : $Q_{\|\|} \approx 0.5$. With \|\| case, only measurement points in the forward scattering zone are contributing. For the $\theta \theta$, a few more measurement points contribute because there is no filtering effect along the $\phi_{s}$ angle.

The analysis made in this section can be used only if the induced current vector $\mathbf{J}_{\mathcal{B}, \mathbf{p}}$ can be considered as collinear with the incident field vector $\mathbf{E}_{\mathcal{B}, \mathbf{p}}^{\mathbf{i}}$. In the following, we use this analysis to interpret the imaging results in the cube case. First of all, to determine if it is in agreement with the assumption of this analysis, we calculated the quantity $\mathcal{A}_{\mathcal{B}, p}$ for the two linear basis (Table V):

$$
\begin{aligned}
& \text { Spherical basis, } \mathcal{A}_{\mathcal{B}, p}=\mathcal{A}_{S p, p}=\frac{1}{N} \frac{1}{N_{s}} \sum_{s} \frac{\left|\mathbf{J}_{\mathcal{B}, \mathbf{p}} \cdot \mathbf{e}_{\mathcal{B}_{(}}^{\mathbf{i}} \mathbf{p}_{8}\right|}{\left\|J_{\mathcal{B}, p}\right\|}
\end{aligned}
$$

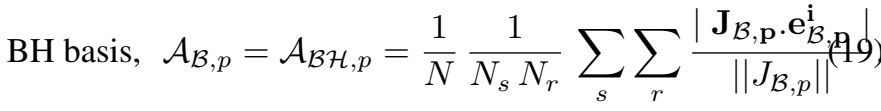

If this value is close to 1 , the image obtained from an element with such an illumination can be interpreted with the following analysis. As it can be seen in Table V, for the cube case, the results from the elements $S_{\phi \phi}, S_{\phi \theta}, S_{\theta \phi}$ and $S_{\theta \theta}$ can be analyzed with the reasoning proposed above, but it is not possible to use it to interpret the results from the elements of the scattering matrix in the $\mathrm{BH}$ basis. With this analysis, the best case should be the $S_{\phi \phi}$ element and then, the $S_{\theta \theta}$ element (see table IV). It's consistent with the imaging results obtained from these elements (see Figures 6, 7, 8 and table I).

\section{Discussion}

If one considers the information contained in the multistatic element of the scattering matrix (see subsection VI A), the copolarized/well-adapted elements seem roughly equivalent. As we have seen in section $\mathrm{V}$, the imaging results from the different elements are not equivalent at all. As a first intuition one might think that the $\mathrm{BH}$ basis should be the best approach and in particular that the $S_{\perp \perp}$ should give the best results as the component of the scattered field is measured colinearly to the incident field vector (see subsection VI B). On the contrary, all the reconstructions from this element failed. This surprising result may be understandable. First, as it can be seen in subsection VI B, even for a small low-contrasted object (the cube), the induced current vectors created in the target are not collinear to the incident field. This can be understood keeping in mind that in the $\mathrm{BH}$ basis, the basis vectors are defined 
in relation to the $\left(\mathbf{k}^{\mathbf{i}}, \mathbf{k}^{\mathbf{s}}\right)$ plane. This implies that with this basis, the polarization selection for source and receiver also plays the role of a filter and its influence can be strong. Thus, for a given illumination, the incident field vector direction is different for each receiver. It implies that for each receiver the induced currents in the target are different. This means that the first step in the inverse algorithm, which is equivalent to a backpropagation process, does not provide a first good estimate of the induced current map. This result is potentially a bad starting point for the algorithm and implies that the algorithm can more easily converge towards a local minimum that is not the global minimum. On the contrary, the co-polarized elements in the spherical basis and the well-adapted elements in the circular basis allow to provide a good estimate of the induced current map at the first step as, for a given illumination, the induced current map is the same for all the receivers. Looking to the differences between the reconstructions with the co-polarized element in linear polarization in the spherical basis $\left(S_{\phi \phi}, S_{\theta \theta}\right)$ and the well-adapted elements in the circular polarization $\left(S_{l r}, S_{r l}\right)$, in the case of a relatively simple target, like the cube, all these elements allow to reconstruct the target with a correct value of the permittivity (Figures 6 - 8). It can be noticed that the reconstruction with the $S_{\theta \theta}$ is anyways worst along the $z$-axis. This makes sense as, in this polarization case, the induced current vectors are nearly collinear to the incident field polarization and the choice of the receiver polarization (along $\mathbf{e}_{\theta}$ ) plays the role of a filter (see section VIB) and also because the polarization vectors of the illuminated field and of the scattered field have no component along the $z$-axis. Now considering the circular helix, with the SVD analysis of the multistatic element (see section VIA), the elements $S_{\phi \phi}, S_{l r}$ and $S_{r l}$ should be better than the $S_{\theta \theta}$, that is indeed the case (see Figures 9 - 12). Based on this analysis, the element $S_{\phi \phi}$ contains a bit more information than the elements $S_{l r}$ and $S_{r l}$, nevertheless the reconstruction with the elements $S_{l r}$ and $S_{r l}$ are a bit better. This can be explain by the geometry of this object, particularly adapted to a circular polarization.

\section{CONCLUSION}

In this study, we have adapted our quantitative imaging procedure from scattering matrix elements in various polarization cases. To focus only on the influence of the polarization, we chose to introduce no a priori information into the inversion procedure (on the searched object or on the noise disturbing the measurements) and no regularization term was added in the cost functional. The scattering matrix was measured for a multistatic configuration in the spherical basis and then, obtained in the other basis by a simple basis change. In this paper, we considered the scattering matrix in different kinds of polarization (linear or circular) and in the linear case, with the two usual basis choices. In each case, the various elements of the scattering matrix were taken as input of our imaging procedure. Even if the different polarization cases are generally considered as equivalent inputs in such non-linear inverse procedures, we have shown that the polarization has a great impact on the reconstructed permittivity maps. The first result is not surprising, i.e., for a given choice of polarization (linear or circular), in a given basis, the co-polarization elements or the well-adapted polarization elements are the best ones. This is in agreement with the analysis of the information content in the multistatic elements of the scattering matrix with a Singular Value Decomposition. Moreover, we have seen that the scattering matrix elements having a similar information content (considering the SVD) can lead to very different reconstructed permittivity maps and thus, a SVD is not sufficient to understand which elements are the optimal ones. We have also shown that a good choice of the basis to describe the polarization is important. For the linear polarization case, results obtained with scattering matrix expressed in the spherical basis are not equivalent to those obtained with Bohren and Huffman basis. This can be understood considering the induced current created inside the target. It can be also noticed that, when one is interested in relatively simple targets, like the cube, even if the imaging results are not similar, almost all the scattering elements in co-polarization (for the linear polarization) or well-adapted polarization (for the circular polarization) allow to reconstruct this object. Looking to more complex objects, as the circular helix which in addition of its shape presents a wire diameter smaller than a third of the wavelength, that is no longer true. For these object, the choice of the polarization (linear or circular), of the basis and of the element of the scattering matrix have an important consequence on the imaging results. In the future, possible combinations of the most relevant scattering matrix elements can help to improve the imaging results according to the studied problem.

\section{ACKNOWLEDGMENTS}

The authors acknowledge the opportunity provided by the Centre Commun de Ressources en Microonde to use its fully equipped anechoic chamber.

The authors want also to thank the Centre de Transfert de Technologie du Mans for the realization of the circular helix.
[1] S. Cloude and E. Pottier, IEEE Trans. on Geosci. Remote Sens. 34, 498 (1996).

[2] W.-M. Boener, Lectures notes POLSARPRO https://earth.esa.int/web/polsarpro/polarimetry-tutorial (2005).
[3] J. Tyo, D. Goldstein, D. Chenault, and J. Shaw, Applied optics 45, 5453 (2006).

[4] M. Mishchenko, L. Travis, and A. Lacis, Scattering, Absorption, and Emission of Light by Small Particles (Cambridge University Press, Cambridge, http://www.giss.nasa.gov/staff/mmishchenko/books.html, 
2002).

[5] M. Garcia, , I. de Erausquin, C. Edmiston, and V. Gruev, Optics Express 23, 14391 (2015).

[6] M. Garcia, C. Edmiston, R. Marinov, A. Vail, and V. Gruev, Optica 4, 1263 (2017).

[7] E. Salomatina-Motts, V. Neel, and A. Yaroslavskaya, Optics and Spectroscopy 107, 884 (2009).

[8] C. Macdonald, U. Tricoli, A. D. Silva, and V. Markel, JOSA A 34, 1330 (2017).

[9] F. Goudail and M. Boffety, JOSA A 33, 9 (2016).

[10] J. V. Zyl and Y. Kim, Synthetic Aperture Radar Polarilmetry (Jet Propulsion Laboratory, 2010).

[11] X. Ma, P. Wu, Y. Wu, and H. Shen, IEEE Journal of selected topics in applied earth observations and remote sensing 11, 743 (2018).

[12] C. Eyraud, R. Vaillon, A. Litman, J.-M. Geffrin, and O. Merchiers, J. Opt. Soc. Am. A 30, 1967 (2013).

[13] X. Ma, P. Wu, Y. Wu, and H. Shen, Journal of Biomedical Optics 7, 359 (2002).

[14] K. Belkebir and M. Saillard, Inverse Problems 21, S1 (2005).

[15] J.-M. Geffrin, P. Sabouroux, and C. Eyraud, Inverse Problems 21, S117 (2005).

[16] A. Litman and L. Crocco, Inverse Problems 26, 020201 (2009).

[17] J.-M. Geffrin and P. Sabouroux, Inverse Problems 25, 024001 (2009).

[18] C. Eyraud, J.-M. Geffrin, and A. Litman, IEEE Trans. Antennas Propag 59 (2011).
[19] G. Bohren and D. Huffman, Absorption and Scattering of Light by Small Particles (John Wiley and Sons, New York, 1983).

[20] B. Toh, R. Cahill, and V. Fusco, IEEE Trans. on Education 46 (2003).

[21] G. Emmons and P. Alexander, Technical Report (1983).

[22] C. Eyraud, A. Litman, A. Hérique, and W. Kofman, Inverse Problems 26, 024005 (2009).

[23] C. Eyraud, J.-M. Geffrin, A. Litman, and J.-P. Spinelli, Radio Science 47 (2012).

[24] A. Litman and K. Belkebir, J. Optical Society of America A 23, 2737 (2006).

[25] H. A. van der Vorst, Cambridge University Press (2003).

[26] B. E. Barrowes, L. F. Teixeira, and J. A. Kong, Microwave and Optical Technology Letters 31, 28 (2001).

[27] O. Merchiers, C. Eyraud, J.-M. Geffrin, R. Vaillon, B. Stout, P. Sabouroux, and B. Lacroix, Optics Express 18, 2056 (2010).

[28] C. Eyraud, J.-M. Geffrin, P. Sabouroux, P. Chaumet, H. Tortel, H. Giovannini, and A.Litman, Radio Science 43, RS4018 (2008).

[29] J.-M. Geffrin, C. Eyraud, A. Litman, and P. Sabouroux, Radio Science 44, RS003837 (2009).

[30] C. Eyraud, J.-M. Geffrin, A. Litman, P. Sabouroux, and H. Giovannini, Applied Physics Letters 89, 244104 (2006).

[31] C. Eyraud, J.-M. Geffrin, A. Litman, and H. Tortel, IEEE Antennas and Wireless Propagation Letters 14 (2015).

[32] H. Saleh, Analogie microonde appliquée à l'étude de la diffraction par des arbres, par des particules atmosphérqiues et des micros-organismes (Phd thesis, Aix Marseille Université, 2017).

[33] O. M. Bucci and T. Isernia, Radio Science 32, 2123 (1997). 